\title{
REMARKS ON $\pi^{-} p$ BACKWARD-SCATTERING EXPERIMENTS
}

A. D. Krisch

University of Michigan

August 6, 1968

In a recent NAL report $D . H$. White has suggested that $\pi^{-} p$ backward scattering should be studied with a system employing a very large gap magnet $(\sim 25$ feet) with its long axis placed parallel to the incident beam. This allows one to observe $d \sigma / d \Omega$ in the approximate range $90^{\circ}<\theta_{\text {c. m. }}<165^{\circ}$. This magnet would probably cost several million dollars. The main justification of the proposed experiment is to study the energy dependence of backward $\pi^{-} p$ scattering.

This note is to suggest that such a procedure will be neither economical nor successful. The $180^{\circ}$ cross section is considerably larger than the $165^{\circ}$ cross section at present energies. There is no question that the $165^{\circ}$ cross section will continue to drop faster as the energy is increased, and the valley between the forward and backward peaks will grow deeper. Thus, explicitly excluding the backward peak from the experiment seems unwise at higher energies.

However, if only the cross section near $180^{\circ}$ is observed in the manner of Frisken et a1., [Phys. Rev. 152, 1162 (1966)] and Kormanyos et al., [Phys. Rev。164, 1661 (1967)] then a much smaller magnet can be used ( 3 feet $\times 1$ foot $\times 1$ foot). This would cost no more than $\$ 50,000$ 。 
The rates with this smaller magnet at $180^{\circ}$ will be higher than with the large magnet placed parallel to the beam.

Moreover, if the energy dependence of the backward scattering is desired, it seems wise to measure backward scattering, not almost backward scattering. 